\title{
Resistance to chemotherapeutic agents and promotion of transforming activity mediated by embryonic stem cell-expressed Ras (ERas) signal in neuroblastoma cells
}

\author{
MINEYOSHI AOYAMA ${ }^{1}$, HIROMI KATAOKA ${ }^{2}$, EIJI KUBOTA ${ }^{2}$, \\ TOYOHIRO TADA ${ }^{3}$ and KIYOFUMI ASAI ${ }^{1}$ \\ ${ }^{1}$ Department of Molecular Neurobiology, ${ }^{2}$ Departments of Gastroenterology and Metabolism, \\ Nagoya City University Graduate School of Medical Sciences; \\ ${ }^{3}$ Nagoya City University School of Nursing, Mizuho-ku, Nagoya 467-8601, Japan
}

Received June 9, 2010; Accepted July 12, 2010

DOI: 10.3892/ijo_00000752

\begin{abstract}
Neuroblastoma is a common childhood tumor derived from neural crest precursor cells. In the present study, we investigated the expression and function of embryonic stem cell-expressed Ras (ERas), a novel Ras family protein previously reported as the specific expression gene in embryonic stem cells (ES cells), in neuroblastoma cell lines. Our results showed that the expressions of ERas were detected in neuroblastoma cell lines by RT-PCR and Western blotting. Therefore, we transfected a full length ERas expression vector into the neuroblastoma cell line SH-SY5Y, which has weak endogenous expression of ERas, and obtained clones with higher levels of expression. Overexpression of ERas did not increase the growth rate of the ERas transfectants but promoted their transforming activity. The ERas transfectants were more resistant to all the chemotherapy agents than the parental cell line. The ability of ERas to rescue cells from the toxic effect of chemotherapeutic agents was inhibited by the phosphatidylinositol 3'-kinase (PI3K) inhibitor PD294002. These results show that the ERas/PI3K pathway may provide resistance to chemotherapy and promote transforming activity in neuroblastoma.
\end{abstract}

\section{Introduction}

Neuroblastoma is one of the most common extracranial solid tumors in children. It is derived from the neural crest and usually arises in the adrenal medulla or a along the

Correspondence to: Dr Mineyoshi Aoyama, Department of Molecular Neurobiology, Nagoya City University Graduate School of Medical Sciences, 1 Kawasumi, Mizuho-cho, Mizuho-ku, Nagoya 467-8601, Japan

E-mail: ao.mine@med.nagoya-cu.ac.jp

Key words: embryonic stem cell-expressed Ras, neuroblastoma, phosphatidylinositol 3'-kinase, drug resistance sympathetic chain (1). The clinical hallmark of neuroblastoma is marked heterogeneity, ranging from subgroups having a very favorable prognosis with a high probability of spontaneous regression to those having a very poor prognosis despite aggressive therapies (2-5). The molecular basis for the difference between neuroblastomas with favorable and poor prognoses is still unclear although some studies have been reported previously (6-13). There are several hypotheses about the pathogenesis of neuroblastoma, but the biological mechanisms governing its unusual behavior have not been elucidated.

The Ras subfamily of small GTPase consists of 13 closely related proteins which can be further divided into five subgroups, each defined by orthologous proteins in C. elegans (14). The first contains the four classical p21 Ras proteins, Harvey-Ras (H-Ras), neuroblastoma-Ras (N-Ras) and two splice variants of Kirsten-Ras (K-Ras 4A and K-Ras 4B). While the oncogenic activity of activated mutants of p21 Ras has been studied extensively, less is known about the oncogenic potential of other members of the family. In neuroblastoma, overexpression of $\mathrm{H}-\mathrm{Ras}$ as well as TrkA is a favorable prognostic factor $(11,15-19)$. It was reported that high expression of H-Ras in neuroblastoma cells is associated with caspase cascade-independent, non-apoptotic programmed cell death (19). There are no reports on the relationship between activated H-Ras mutant genes and the tumorigenesis of neuroblastoma. Recently, Takahashi et al described embryonic stem cell-expressed Ras (ERas), a member of the Ras protein family not known previously. Northern blot analyses detected a single ERas transcript only in undifferentiated mouse embryonic stem cells (ES cells). ERas was not detected in differentiated ES cells or in 12 adult mouse somatic tissues. Thus, the specific expression of ERas is a common property of ES cells. The transforming oncogene ERas is important in the tumor-like growth properties of ES cells (20).

One-third of all solid tumors have activated mutants of classical p21 Ras genes (21), but the relationship between activated mutants of p21 Ras and the tumorigenesis of neuroblastoma is not clear. This raises the possibility that other members of the Ras subfamily may play roles in the 
malignancy of neuroblastomas. Therefore, we investigated the expression of ERas, a novel member of the Ras protein family, in neuroblastoma cells and found that the expression of ERas in neuroblastoma cells results in the promotion of transforming activity and resistance to death from cytotoxic agents.

\section{Materials and methods}

Cell culture and transfection. Human neuroblastoma cells (SAN, KAN, NLF, KCN, IMR32, SH-SY5Y, GOTO, TGW, Nagai, SKN-DZ) were maintained at $37^{\circ} \mathrm{C}$ in an atmosphere of $5 \% \mathrm{CO}_{2}$ in the air in RPMI-1640 medium supplemented with $10 \%$ fetal bovine serum (FBS), $100 \mathrm{IU} / \mathrm{ml}$ penicillin, and $100 \mu \mathrm{g} / \mathrm{ml}$ streptomycin. Stable transfections of SH-SY5Y cells were performed with the empty plasmid pcCAG-IP, gift from Dr H. Niwa (22) or with the expression plasmid for ERas, pCAG-hERas, generously provided by Dr S. Yamanaka, as previously described (20), using Lipofectamine 2000 transfection reagent according to the manufacturer's instructions (Life Technologies, Gaithersburg, MD). The transfected cells were cultured in the presence of $2 \mu \mathrm{g} / \mathrm{ml}$ of puromycin (Sigma Chemical Co., St. Louis, MO). The puromycin-resistant cells were subcloned as single-cell clones. The expression of ERas protein by the single-cell clones was characterized by Western blotting.

Isolation of total RNA and reverse transcriptase polymerase chain reaction $(R T-P C R)$. Total RNA was prepared using TRIzol reagent (Invitrogen, Carlsbad, CA). Reverse transcription was carried out using random primers and SuperScript II RNase $\mathrm{H}^{-}$reverse transcriptase (Invitrogen). The resultant cDNA was subjected to PCR-based amplification. Oligonucleotides used to amplify ERas and $B$-actin mRNAs were as follows: ERas: forward, 5'-TCCCCTGTTGTCTTGCT TCT-3', reverse, 5'-TGTCCAGGGTCAACTCCTTC-3'; and ß-actin: forward, 5'-GACCTGACTGACTACCTCAT-3', reverse, 5'-AGCAAGCAGGAGTAGACGA-3'. Amplified products were separated electrophoretically on agarose gels and visualized by ethidium bromide staining. The gels were photographed under ultraviolet illumination.

Immunoblotting. Cells were washed in PBS, centrifuged, and then lysed on ice for $30 \mathrm{~min}$ in Triton lysis buffer containing 25 mM Tris- $\mathrm{HCl}$ (pH 8.0), $137 \mathrm{mM} \mathrm{NaCl}, 2.7 \mathrm{mM} \mathrm{KCl}, 1 \%$ Triton X-100 and protease inhibitor cocktail (Sigma). Protein concentrations were determined using a BCA protein assay kit (Pierce Biotechnology, Rockford, IL). Protein samples were resolved by $12 \%$ SDS-PAGE, and electrotransferred onto PVDF membranes (Immobilon-P, Millipore, Billerica, MA) at $180 \mathrm{~mA}, 100 \mathrm{~V}$ for $45 \mathrm{~min}$ at $4^{\circ} \mathrm{C}$. The membrane filters were blocked for $1 \mathrm{~h}$ with $5 \%$ powdered milk in TBS-T $(0.1 \%$ Tween-20, $20 \mathrm{mM}$ Tris- $\mathrm{HCl}, 137 \mathrm{mM} \mathrm{NaCl}, \mathrm{pH}$ 7.6) and then incubated with rabbit anti-ERas (20) diluted 1:500, rabbit anti-Akt (Cell Signaling) diluted 1:500, rabbit anti-phosphoAkt (Cell Signaling) diluted 1:500, rabbit anti-p44/p42 (Cell Signaling) diluted 1:500, or rabbit anti-phospho-p44/p42 (Cell Signaling) diluted 1:500 in TBS-T. The blots were developed using anti-rabbit $\mathrm{IgG}$ conjugated to horseradish peroxidase (1:5000 dilution, Amersham Biosciences Corp.,
Piscataway, NJ), and bands were visualized with an enhanced chemiluminescence method (ECL, Amersham Biosciences Corp.) according to the manufacturer's instructions. For normalization of protein loading, blots were stripped and reprobed with a polyclonal anti-actin antibody (20-33; Sigma Chemical Co.).

Cell proliferation and soft agar assay. Cells were seeded in triplicate wells of 24 -well plates $\left(5 \times 10^{3}\right.$ cells per well) in culture medium containing $10 \%$ FBS and were allowed to adhere to the cell culture dish for $24 \mathrm{~h}$. At the indicated times, cell numbers were assessed using the MTT assay (Cell Counting Kit 5, Dojindo, Kumamoto, Japan) according to the manufacturer's protocol. For soft agar assays, $2.5 \times 10^{3}$ cells of the stable transfectants or the parental SH-SY5Y cells were seeded in triplicate in $35-\mathrm{mm}$ cell culture plates containing $0.2 \%$ agar and RPMI-1640 medium supplemented with $10 \%$ FBS and colonies were counted after 21 days.

Cytotoxicity assay. SH-SY5Y (parental cells), CAG-6 and CAG-9 (control vector-transfected cells), and ERas-5 and ERas-7 (ERas-transfected cells) in RPMI-1640 containing $10 \%$ FBS were seeded in 96-well plates at a density of $1 \times 10^{4}$ cells/well. All experiments were performed in triplicate and repeated at least three times. Two days after replating the cells were treated with cisplatin $(3 \mu \mathrm{g} / \mathrm{ml})$, doxorubicin $(0.5 \mu \mathrm{g} / \mathrm{ml})$, vinblastine $(2.5 \mathrm{ng} / \mathrm{ml})$, or etoposide $(3 \mu \mathrm{g} / \mathrm{ml})$ for $48 \mathrm{~h}$. For combination treatments, LY294002 (LY; $20 \mu \mathrm{M}$ ), an inhibitor of the PI3K signaling pathway, was added $30 \mathrm{~min}$ before the cytotoxic agents. A colorimetric MTT assay was used to measure cell survival.

Statistical analysis. The results of the MTT and soft agar assays were analyzed by an unpaired Student's t-test. Differences with p-values $<0.01$ were considered significant.

\section{Results}

Expression of ERas in neuroblastoma cell lines. Expression of ERas in neuroblastoma cell lines was assessed by RT-PCR. ERas expression was observed in at least six of ten cell lines (SAN, Nagai, KCN, IMR-32, SKN-DZ, and SH-SY5Y) and the other four cell lines had faint or no expression of ERas (Fig. 1). To avoid contamination by genomic DNA, the sample RNA without the reverse transcription reaction was subjected to PCR analysis and no bands were detected in any of the samples. To confirm ERas expression, Western blotting was performed with three cell lines. ERas protein was detectable in IMR-32, weakly in SH-SY5Y, and not in GOTO, which was in accordance with RT-PCR results.

Activation of PI3K pathway by ERas expression in ERas transfectants. To address the question whether ERas can be relative with the tumor formation of neuroblastoma cells, we transfected the expression plasmid for ERas or the empty plasmid into SH-SY5Y neuroblastoma cells, which expressed endogenous ERas weakly, and established two stable transfectants with higher expression of ERas (named ERas-5 and ERas-7). As shown in the Western blots in Fig. 2A, the levels of ERas were higher in ERas-5 and ERas-7 cells than in the 
A

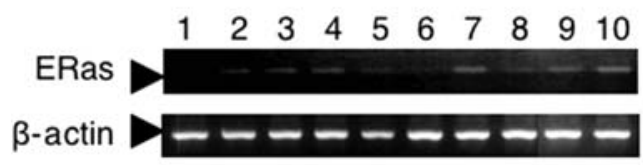

RT (-)
ERas

B

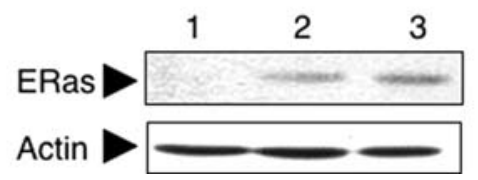

Figure 1. Expression of ERas in human neuroblastoma cell lines. (A) Total RNA prepared from the cultured cells was analyzed by RT-PCR. Expression of $\beta$-actin served as an internal control. As a negative control, RNA without reverse transcription was subjected to PCR analysis. Lane 1, GOTO; lane 2, KAN; lane 3, SAN; lane 4, Nagai; lane 5, KCN; lane 6, NLF; lane 7, IMR-32; lane 8, TGW; lane 9, SKN-DZ; lane 10, SH-SY5Y. Lane 11 is the sample from SH-SY5Y subjected to reverse transcription and the PCR reaction. (B) Lysates containing $40 \mu \mathrm{g}$ of protein from 3 neuroblastoma cell lines were analyzed for ERas by Western blotting; lane 1, GOTO; lane 2, SH-SY5Y; lane 3, IMR-32. For normalization of protein loading, blots were stripped and reprobed with a polyclonal anti-actin antibody (20-33; Sigma Chemical Co.).

A

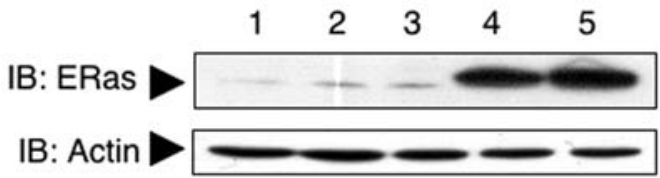

B

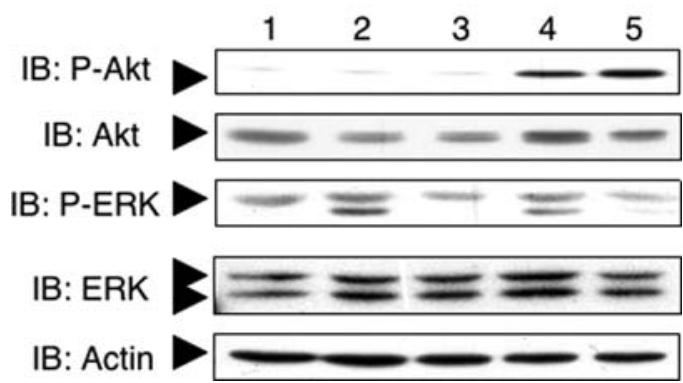

Figure 2. Activation of ERas signal transduction pathways. (A) Expression of ERas protein in SH-SY5Y and its transfectants. (B) Lysates containing $40 \mu \mathrm{g}$ of lysate protein was analyzed for P-Ser-Akt, total Akt, P-Thr/TyrERK and total ERK by Western blot analysis. Lane 1, parental SH-SY5Y; lanes 2 and 3, CAG-6 and CAG-9, control-transfectants; lanes 4 and 5, ERas-5 and ERas-7, ERas-transfectants.

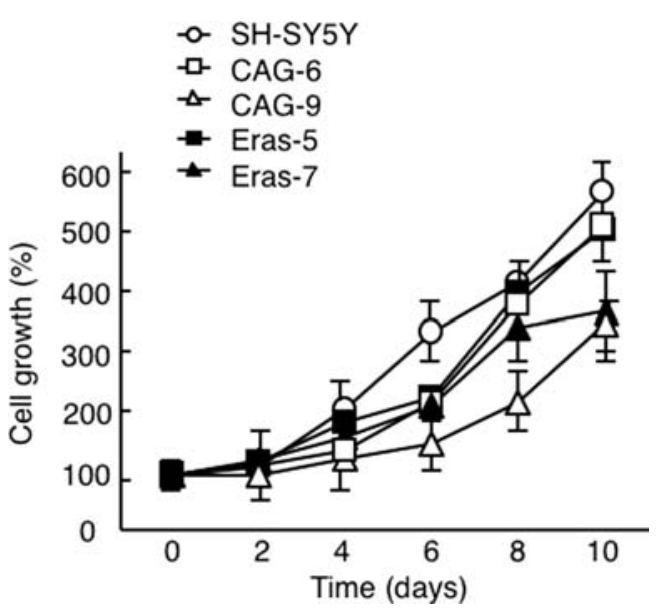

Figure 3. Effects of ERas overexpression on growth of SH-SY5Y. SH-SY5Y and the indicated transfectants were grown in medium containing $10 \%$ FBS. Cells were harvested every $48 \mathrm{~h}$ and their numbers were determined by MTT assay in triplicate. The data represent the means \pm SE from three independent experiments.

parental SH-SY5Y cells and the control transfectants (CAG-6 and CAG-9). The blots were reprobed with anti-actin antibody to confirm equal loading of cell lysates. No obvious morphological changes were seen in SH-SY5Y cells overexpressing ERas (data not shown).

Akt was reported previously to be a downstream effector of the ERas/PI3K pathway, though ERas did not stimulate the MAPK pathway (20). To determine whether the PI3K pathway is specifically stimulated by ERas expression without stimulation of the MAPK pathway in neuroblastoma cells, we assayed PI3K activity by Western blots using a phosphoAKT antibody that specifically recognizes activated AKT. Blots were reprobed with an anti-AKT antibody to ensure equal protein loading in each lane. Phosphorylated AKT was detected strongly in ERas-5 and ERas-7 cells and weakly in parental SH-SY5Y and control cells (Fig. 2B). We also examined the effect of ERas on the MAPK pathway by examining the phosphorylation of ERK by Western blotting. Phosphorylated ERK was detected in all cells tested, and there was no difference with or without ERas overexpression.

Relationship between overexpression of ERas and growth rate and transforming activity of neuroblastoma cells. The growth rates of each transfectants under optimal culture conditions (10\% serum) were determined. As shown in Fig. 3, overexpression of ERas had no significant effect on the growth rate of cells. To examine whether the ERas transfectants could grow in soft agar medium, each transfectant was cultured in soft agar medium for three weeks and the number of colonies formed by each transfectant was counted. ERas-5 and ERas-7 cells formed large distinct colonies in significantly greater numbers compared to vector-transfected cells and the parental SH-SY5Y cells (Fig. 4).

Effect of ERas expression on survival of SH-SY5Y cells treated with chemotherapeutic agents. We analyzed the effects of ERas expression on the response of neuroblastoma 
A

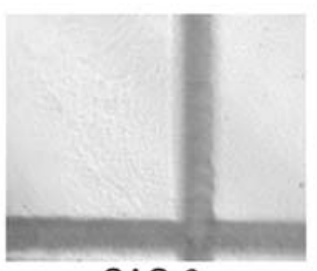

CAG-6
ERas-7

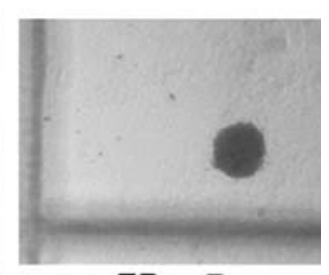

B

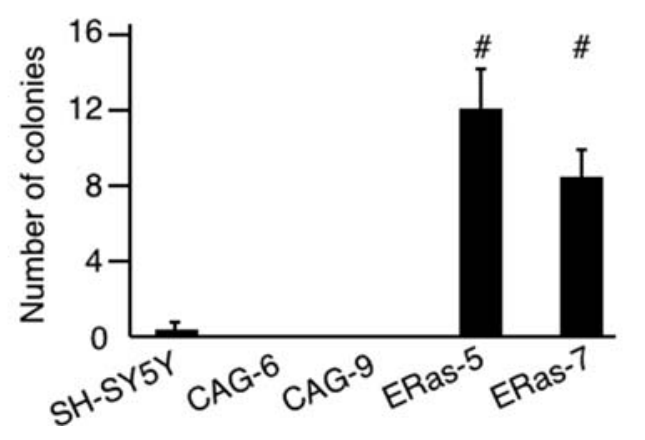

Figure 4. Anchorage-independent growth of ERas-overexpressing transfectants. The parental SH-SY5Y cells and the indicated transfectants $\left(2.5 \times 10^{3}\right.$ cells/dish) were grown in soft agar medium. After three weeks of culture, cells were examined by phase-contrast microscopy (upper), and the numbers of colonies were counted (lower). Data are the means \pm SE from three independent experiments. ${ }^{*} \mathrm{p}<0.05$, compared to parental SH-SY5Y.

cells to chemotherapy in vitro. Four chemotherapeutic agents commonly used to treat neuroblastoma, cisplatin, doxorubicin, vinblastine, and etoposide, were used. Cell survival was determined by the MTT assay. All four chemotherapeutic agents caused death of SH-SY5Y, and LY294002, a selective inhibitor of PI3K, significantly decreased the survival rate of SH-SY5Y (Fig. 5A). To determine whether overexpression of ERas would increase the survival of SH-SY5Y, the survival rate of each transfectant after treatment with chemotherapeutic agents was assayed. The survival rates of the ERas transfectants were significantly higher than that of the parental SH-SY5Y cells and the control transfectants after treatment with all four chemotherapeutic agents (Fig. 5B). To identify the signal transduction pathway responsible for protecting the cells, we assessed the effect of ERas signal transduction on neuroblastoma cell survival in the presence of the PI3K inhibitor, LY294002. The inhibitor was added to the parental SH-SY5Y cells, the control transfectants and the ERas transfectants $30 \mathrm{~min}$ before adding of chemotherapeutic agents and cell numbers were assessed $48 \mathrm{~h}$ later. Treatment of ERas transfectants with chemotherapeutic agents and the PI3K inhibitor LY294002 decreased cell survival. The survival rates were not significantly different between the ERas transfectants and the parental SH-SY5Y cells or the control transfectants. The protective effect of ERas against the four chemotherapeutic agents was blocked by LY294002 (Fig. 5C).
A

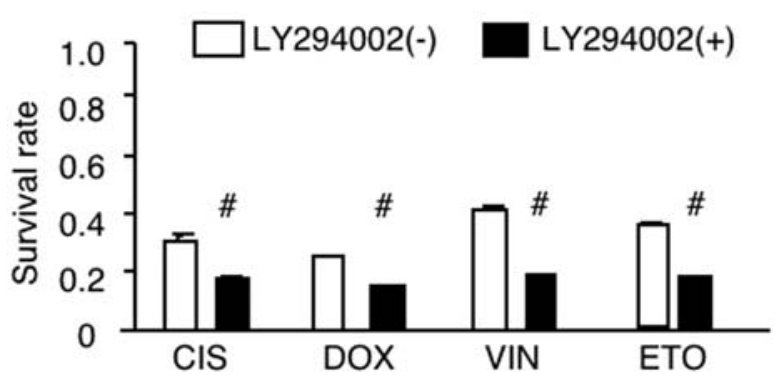

B

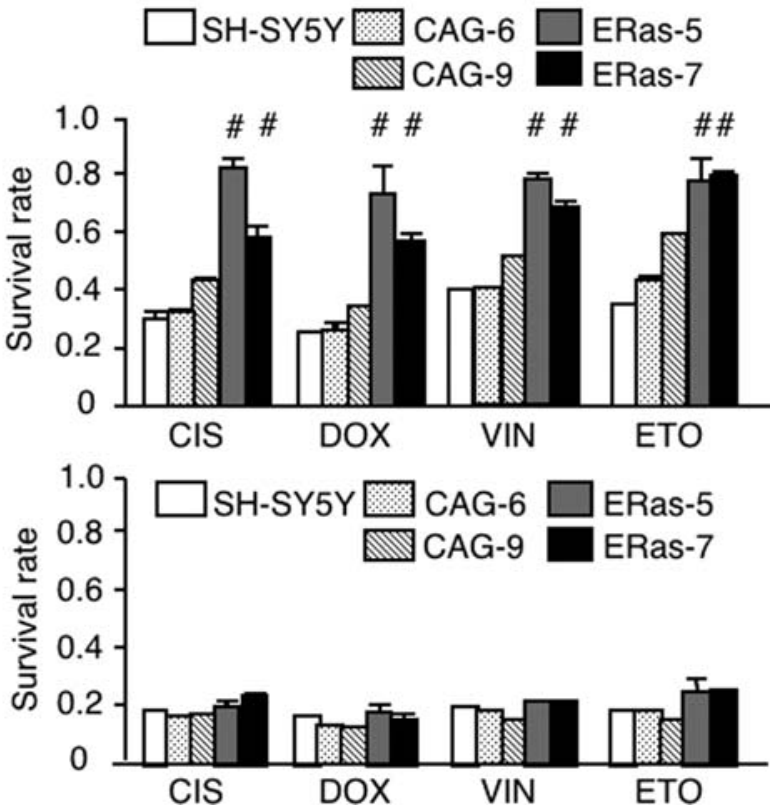

Figure 5. Effect of ERas expression on chemoresistance of neuroblastoma cells. (A) SH-SY5Y cells were cultured in medium containing $10 \%$ FBS in 96-well plates and pretreated without (white bar) or with (black bar) LY294002 (LY; $20 \mu \mathrm{M}$ ), an inhibitor of the PI3K signaling pathway, 30 min before addition of cisplatin (CIS; $3 \mu \mathrm{g} / \mathrm{ml}$ ), doxorubicin (DOX; $0.5 \mu \mathrm{g} / \mathrm{ml}$ ), vinblastine (VIN; $2.5 \mu \mathrm{g} / \mathrm{ml}$ ), or etoposide (ETO; $3 \mu \mathrm{g} / \mathrm{ml}$ ). After $48 \mathrm{~h}$, cell survival rates were determined by MTT assay. Data are the means of triplicate samples (error bars, \pm SD) and represent the rate of cells surviving compared to untreated cells. Representative results are shown from one of at least three separate experiments. The differences in survival rates between SH-SY5Y with or without inhibitor treatments were significant $(\mathrm{p}<0.01$ for all cases). (B) SH-SY5Y cells and ERas transfectants were cultured in medium containing $10 \%$ FBS in 96-well plates and treated with each chemotherapeutic agent for $48 \mathrm{~h}$. Cells were assayed as described in (A). The differences in survival rates between SH-SY5Y and either ERas-5 or ERas-7 were significant ( $\mathrm{p}<0.01$ for all cases). (C) SH-SY5Y cells and ERas transfectants were cultured in medium containing 10\% FBS in 96-well plates and pretreated with LY294002 (LY; $20 \mu \mathrm{M}) 30$ min before addition of each chemotherapeutic agent, and $48 \mathrm{~h}$ later cells were assayed as described in (A). The differences in survival rates between SH-SY5Y and either ERas-5 or ERas-7 were not significant in any of the cases. ${ }^{*} \mathrm{p}<0.01$, compared to parental SH-SY5Y.

\section{Discussion}

The finding that human neuroblastomas express ERas, a novel member of the Ras protein family, suggests an important role for ERas in neuroblastoma development. Recently, a Japanese group has reported ERas mRNA expression in some cell lines of colorectal cancer, pancreatic cancer and breast cancer, revealing that the ERas gene transcription was regulated by epigenetic silencing (23). A still earlier report (24) 
showed that the ERas gene was located on the X chromosome and it was identified as a pseudogene not expressed in any tissues, including ES cells. To confirm the specificity of expression, we used specific rabbit antiserum against ERas and showed the presence of ERas in tumor cells. Using RT-PCR, ERas expression was observed in at least six of ten neuroblastoma cell lines. Thus, we confirmed the expression of ERas in neuroblastoma cell lines.

The signaling pathway downstream of ERas responsible for generating the phenotypes we observed was suggested to be the PI3K pathway, not the mitogen-activated protein kinase (MAPK) pathway. As reported previously, ERas could not bind to Raf or activate the MAPK pathway (20). In this study, we did not observe activation of the MAPK cascade by ERas expression. The SH-SY5Y cell line expresses ERas endogenously but the levels are only faintly detectable by Western blotting. Also, phosphorylation of the signaling protein AKT was detected and overexpression of ERas, reported to be a constitutive active protein, increased phosphorylated AKT. However, ERas expression did not alter the amount of phosphorylated ERK, a downstream effector of the MAPK pathway.

Our studies on the role of ERas in cell proliferation showed that overexpression of ERas did not significantly increase the rate of proliferation of ERas transfectants compared to the parental SH-SY5Y cells or the control transfectants. As reported previously, only small changes in the cell cycle were observed in ERas-null cells compared to wild-type cells. It has been suggested that PI3K may have a growth-promoting effect that is independent of cell cycle control (20). Generally, tumor cells have the potential for higher proliferation, so significant promotion of growth rate by ERas may not be observed. With the soft-agar colonyformation assay, ERas transfectants had significantly increased colony formation compared to the parental SH-SY5Y cells or the control transfectants, which is in accordance with a previous report (20). The role of ERas as an oncogenic factor in neuroblastoma is supported by the anchorage-independent growth of neuroblastoma cells in soft-agar.

We demonstrated that the ERas-Akt pathway was activated more in SH-SY5Y clones after transfection. Steady-state phosphorylation of Akt by ERas was observed in the ERas transfectants. Furthermore, there was increased resistance to chemotherapeutic agents in the ERas transfectants compared to the parental SH-SY5Y cells or the control transfectants. When combined with chemotherapy, the PI3K-specific inhibitor LY294002 decreased cell survival toward the baseline compared to chemotherapy alone. Thus, the activated ERas-Akt pathway probably plays an important role in the increased chemoresistance observed clinically in ERas-expressing tumors.

In conclusion, this study demonstrates that neuroblastoma cells express ERas protein. ERas expression promotes tumorigenicity and the chemoresistance. Targeting the ERas/Akt pathway may be a useful therapeutic approach for treating chemoresistant tumors.

\section{Acknowledgements}

We thank Dr Shinya Yamanaka (Kyoto University, Institute for Frontier Medical Sciences, Department of Stem Cell
Biology) for helpful advice and for providing the anti-ERas antibody and plasmid for ERas. We thank Ms. Manami Yamamoto for excellent technical assistance. This study was supported by a Grant-in-Aid for Scientific Research on Priority Areas, a Grant-in-Aid for Scientific Research (C), and a Grant-in Aid for Young Scientists (B) from the Ministry of Education, Culture, Sports, Science and Technology, Japan.

\section{References}

1. Brodeur GM, Pritchard J, Berthold F, et al: Revisions of the international criteria for neuroblastoma diagnosis, staging, and response to treatment. J Clin Oncol 11: 1466-1477, 1993.

2. Pritchard J and Hickman JA: Why does stage 4s neuroblastoma regress spontaneously? Lancet 344: 869-870, 1994.

3. Yamamoto K, Hanada R, Kikuchi A, et al: Spontaneous regression of localized neuroblastoma detected by mass screening. J Clin Oncol 16: 1265-1269, 1998.

4. Brodeur GM: Molecular basis for heterogeneity in human neuroblastomas. Eur J Cancer 31: 505-510, 1995.

5. Brodeur GM and Nakagawara A: Molecular basis of clinical heterogeneity in neuroblastoma. Am J Pediatr Hematol Oncol 14: 111-116, 1992

6. Ohira M, Morohashi A, Inuzuka H, et al: Expression profiling and characterization of 4200 genes cloned from primary neuroblastomas: identification of 305 genes differentially expressed between favorable and unfavorable subsets. Oncogene 22: 5525-5536, 2003

7. Brodeur GM, Azar C, Brother M, et al: Effect of genetic factors on prognosis and treatment. Cancer 70: 1685-1694, 1992.

8. Brodeur GM, Seeger RC, Schwab M, Varmus HE and Bishop JM: Amplification of $\mathrm{N}$-myc in untreated human neuroblastomas correlates with advanced disease stage. Science 224: 1121-1124, 1984.

9. Seeger RC, Brodeur GM, Sather H, et al: Association of multiple copies of the N-myc oncogene with rapid progression of neuroblastomas. N Engl J Med 313: 1111-1116, 1985.

10. Nakagawara A, Arima M, Azar CG, Scavarda NJ and Brodeur GM: Inverse relationship between trk expression and $\mathrm{N}$-myc amplification in human neuroblastomas. Cancer Res 52: $1364-1368,1992$

11. Nakagawara A, Arima-Nakagawara M, Scavarda NJ, Azar CG, Cantor AB and Brodeur GM: Association between high levels of expression of the TRK gene and favorable outcome in human neuroblastoma. N Engl J Med 328: 847-854, 1993.

12. Gross N, Beretta C, Peruisseau G, Jackson D, Simmons D and Beck D: CD44H expression by human neuroblastoma cells: relation to MYCN amplification and lineage differentiation. Cancer Res 54: 4238-4242, 1994.

13. Berwanger B, Hartmann O, Bergmann E, et al: Loss of a FYN-regulated differentiation and growth arrest pathway in advanced stage neuroblastoma. Cancer Cell 2: 377-386, 2002.

14. Ehrhardt A, Ehrhardt GR, Guo X and Schrader JW: Ras and relatives - job sharing and networking keep an old family together. Exp Hematol 30: 1089-1106, 2002.

15. Tanaka T, Slamon DJ, Shimada H, et al: A significant association of Ha-ras p21 in neuroblastoma cells with patient prognosis. A retrospective study of 103 cases. Cancer 68: 1296-1302, 1991

16. Tanaka T, Sugimoto T and Sawada T: Prognostic discrimination among neuroblastomas according to Ha-ras/trk A gene expression: a comparison of the profiles of neuroblastomas detected clinically and those detected through mass screening. Cancer 83: 1626-1633, 1998.

17. Matsunaga T, Takahashi H, Ohnuma N, et al: Expression of $\mathrm{N}$-myc and c-src protooncogenes correlating to the undifferentiated phenotype and prognosis of primary neuroblastomas. Cancer Res 51: 3148-3152, 1991.

18. Nakada K, Fujioka T, Kitagawa H, Takakuwa T and Yamate N: Expressions of $\mathrm{N}$-myc and ras oncogene products in neuroblastoma and their correlations with prognosis. Jpn J Clin Oncol 23: 149-155, 1993

19. Kitanaka C, Kato K, Ijiri R, et al: Increased Ras expression and caspase-independent neuroblastoma cell death: possible mechanism of spontaneous neuroblastoma regression. J Natl Cancer Inst 94: 358-368, 2002. 
20. Takahashi K, Mitsui K and Yamanaka S: Role of ERas in promoting tumour-like properties in mouse embryonic stem cells. Nature 423: 541-545, 2003

21. Kiaris H and Spandidos DA: Mutations of ras genes in human tumours (Review). Int J Oncol 7: 413-421, 1995.

22. Niwa H, Masui S, Chambers I, Smith AG and Miyazaki J: Phenotypic complementation establishes requirements for specific POU domain and generic transactivation function of Oct-3/4 in embryonic stem cells. Mol Cell Biol 22: 1526-1536, 2002.
23. Yasuda K, Yashiro M, Sawada T, Ohira M and Hirakawa K: ERas oncogene expression and epigenetic regulation by histone acetylation in human cancer cells. Anticancer Res 27: 4071-4075, 2007.

24. Miyoshi J, Kagimoto M, Soeda E and Sakaki Y: The human c-Ha-ras2 is a processed pseudogene inactivated by numerous base substitutions. Nucleic Acids Res 12: 1821-1828, 1984. 